\title{
SIR Model with the Person Trip to Analyze the Epidemic of Influenza
}

\author{
Ryo Sakamaki ${ }^{\mathrm{a},}$, Satoru Fujita ${ }^{\mathrm{a}}$ \\ ${ }^{a}$ Graduate School of Computer and Information Sciences, Hosei University, Japan
}

*Corresponding Author: 19t0008@ cis.k.hosei.ac.jp

\begin{abstract}
This study proposes an epidemic model with the person trip and analyzes real epidemic of influenza virus in Kanto region in Japan from 2017 to 2018. Based on the SIR model with vaccination, we obtain an epidemic model and then divide the single-epidemic region into multiple groups, which ensure various infections with the person trip. Next, the next-generation matrix is constructed to calculate both the basic reproduction number and the reproduction number for each group. Then, using the record of the number of infected people from the 40th week in 2017 to the 22nd week in 2018, we estimate the parameter values of the infection and recovery rates. Finally, we discuss the suppression of epidemics by applying the estimated parameter values and the vaccine supply data. The basic reproduction number is obtained to be 1.08 as a consequence of the parameters' estimation and calculation. If adequate vaccinations were taken by individuals to prevent infection, it can suppress the number of the infected people by about $30 \%$ at the peak time while the total number of infected people can be suppressed by about $15 \%$.
\end{abstract}

Keywords: SIR model, Epidemic model, Person trip, Influenza, Reproduction number.

\section{Introduction}

The threats of epidemic diseases are increasing and becoming more serious problems since the year 2000. For example, the outbreak of severe acute respiratory syndrome (SARS) in southern China in 2003 resulted in the death of about 774 people. In addition, the influenza virus (H1N1) that spread worldwide in 2009 gave an imminent warning to the public. In particular, the influenza virus infects numerous people yearly. This is due to the antigenic drift observed in the virus, and the recent growth of the population enhances the spread of the virus. Therefore, the development of preventive measures against the epidemic is now considered a more important issue to be addressed urgently from the perspective of public health.

The application of the SIR model is a typical way of analyzing an epidemic ${ }^{(1)}$. It classifies the people in the epidemic region into three compartments: susceptible, infectious, and recovered; it expresses the spread of the epidemic process as simultaneous differential equations. During the epidemics of Ebola virus in West Africa from 2014 to 2015, an extension of the SIR model was actually applied to the epidemic analysis for suppressing the spread of the disease ${ }^{(2)}$. Moreover, there were assumptions that the epidemic regions were regarded as a single group and the infected persons were located evenly in the area. Consequently, the people close to each other and the people far from each other had the same possibility of being infected, so there were no differences regarding the distance between them. Additionally, the analysis did not consider the population movement around the area. For example, urban areas in Japan have numerous people coming and going across the areas during the day and in the night, so that the occurrence of infection may differ depending on time and place. This study formulates an epidemic model that divides the epidemic region into multiple groups by considering the person trip. Furthermore, this model is applied to analyze the real epidemic of influenza virus in the Kanto region in Japan from 2017 to 2018 and discuss how much the spread of the epidemic can be suppressed.

\section{The Epidemic Model}

This section formulates an extension of the SIR model with the person trip. First, we describe the KermackMcKendrick SIR model, which is the basis of our epidemic model. This SIR model classifies residents of an epidemic region into three compartments: susceptible, infectious, and recovered. It applies simultaneous differential equations to express the transitions between the compartments. Let $S, I$ 
and $R$ denote the number of susceptible, infectious, and recovered people, respectively. The difference between $S$ and $I$ is proportional to the number of the susceptible and the infectious people in contact. Moreover, the difference between $I$ and $R$ is proportional to the number of the infectious. Therefore, the differences of $S, I$ and $R$ are expressed as the following equations:

$$
\begin{gathered}
\frac{d S}{d t}=-\beta S \frac{I}{N} \\
\frac{d I}{d t}=\beta S \frac{I}{N}-\gamma I \\
\frac{d R}{d t}=\gamma I
\end{gathered}
$$

where $N, \beta$, and $\gamma$ denote the number of the contact $(S+I+R)$, infection rate, and recovery rate, respectively.

To solve these problems using the SIR model, we divide the epidemic region into $n$ groups and define $S_{i}, I_{i}$, and $R_{i}$ as the susceptible, infectious, and recovered people of group $i$, respectively. In addition, we assume that the model enables different infections with the person trip. Let $\beta_{i}, \gamma_{i}$, $N_{i}, T_{i, j}$, and $N_{j}^{d}$ denote the infection rate of group $i$, the recovery rate of group $i$, the number of the contact of group $i\left(S_{i}+I_{i}+R_{i}\right)$, the transfer rate from group $i$ to $j$, and the number of the contact of daytime group $j\left(\sum_{k=1}^{n} T_{k, j} N_{k}\right)$. First, we investigate the infection in the daytime for the susceptible in group $i$. In the daytime, the number of the susceptible which moves to group 1 is given by $T_{\mathrm{i}, 1} S_{i}$, while the number of the infectious which moves to group 1 is given by $\sum_{k=1}^{n} T_{k, 1} I_{k}$. Similarly, the number of contacts in the daytime is given by $\sum_{\mathrm{k}=1}^{n} T_{\mathrm{k}, 1} N_{k}$. Thus, if they are infected with the same infection rate at each group, then the occurrence of infection that are susceptible in group $i$ moves to group 1 in the daytime is given by the following equation:

$$
\lambda_{i, 1}^{d}=\frac{T_{i, 1} S_{i}}{N_{1}^{d}} \sum_{k=1}^{n} \beta_{k} T_{k, 1} I_{k}
$$

Moreover, the occurrence of infection that is susceptible in group $i$ moves to group $n$ in the daytime is expressed as the equation below:

$$
\lambda_{i, n}^{d}=\frac{T_{i, n} S_{i}}{N_{n}^{d}} \sum_{k=1}^{n} \beta_{k} T_{k, n} I_{k}
$$

The finite sum from $\lambda_{i, 1}^{d}$ to $\lambda_{i, n}^{d}$ is the total occurrence of the infection for group $i$ susceptible in the daytime can be given by the following equation:

$$
\lambda_{i}^{d}=\sum_{j=1}^{n} \lambda_{i, j}^{d}=S_{i} \sum_{j=1}^{n} \frac{T_{i, j}}{N_{j}^{d}} \sum_{k=1}^{n} \beta_{k} T_{k, j} I_{k}
$$

Next, we examine the infection at night time for group $i$. The infection occurs in the same group if there is no movement at night. Thus, we obtain the following equation:

$$
\lambda_{i}^{n}=\beta_{i} S_{i} \frac{I_{i}}{N_{i}}
$$

From equations (4) and (5), we obtain equation (6) which demonstrates the occurrence of the infection for the susceptible in group $i$ :

$$
\begin{aligned}
\lambda_{i} & =\frac{1}{2}\left(\lambda_{i}^{d}+\lambda_{i}^{n}\right) \\
& =\frac{1}{2}\left(S_{i} \sum_{j=1}^{n} \frac{T_{i, j}}{N_{j}^{d}} \sum_{k=1}^{n} \beta_{k} T_{k, j} I_{k}+\beta_{i} S_{i} \frac{I_{i}}{N_{i}}\right)
\end{aligned}
$$

To ensure consistency in view of the SIR model, the equation is multiplied by $1 / 2$ with the identity matrix $\mathrm{T}$ that denotes no movement. Moreover, since the recovery is not based on movements, we obtain equation (7) that expresses the amount of recovery for the infectious in group $i$ :

$$
\sigma_{i}=\gamma_{i} I_{i}
$$

Finally, we examine the vaccination effect for group $i$. Generally, the acquisition of immunity from vaccination is not $100 \%$, and vaccination has a period in which it is performed. Consequently, let $p$ and $v_{i}(t)$ denote the disease prevention rate by vaccination and vaccination rate based on the effective period, respectively. Thus, we get equation (8), which expresses the vaccination for the susceptible in group $i$ :

$$
\begin{aligned}
\mu_{i} & =p v_{i}(t) S_{i} \\
v_{i}(t) & =\left\{\begin{array}{l}
v_{i}\left(t_{0} \leq t \leq t_{1}\right) \\
0 \text { (otherwise) }
\end{array}\right.
\end{aligned}
$$

where $t_{0}$ and $t_{1}$ denote the start time and the end time of vaccination. By applying equations (4), (7), and (8), we get the following SIR model with person trip:

$$
\begin{gathered}
\frac{d S_{i}}{d t}=-\lambda_{\mathrm{i}}-\mu_{i} \\
\frac{d I_{i}}{d t}=\lambda_{\mathrm{i}}-\sigma_{i} \\
\frac{d R_{i}}{d t}=\sigma_{i}+\mu_{i} \\
\lambda_{i}=\frac{1}{2}\left(\lambda_{i}^{d}+\lambda_{i}^{n}\right) \\
\lambda_{i}^{d}=S_{i} \sum_{j=1}^{n} \frac{T_{i, j}}{N_{j}^{d}} \sum_{k=1}^{n} \beta_{k} T_{k, j} I_{k} \\
\lambda_{i}^{n}=\beta_{i} S_{i} \frac{I_{i}}{N_{i}} \\
\sigma_{i}=\gamma_{i} I_{i}
\end{gathered}
$$




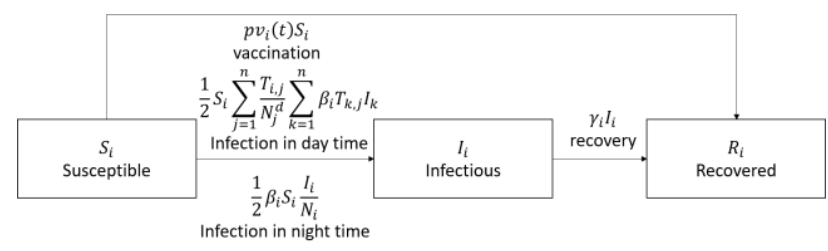

Fig. 1. The transition in the model.

$$
\begin{gathered}
\mu_{i}=p v_{i}(t) S_{i} \\
v_{i}=\left\{\begin{array}{c}
v_{i}\left(t_{0} \leq t \leq t_{1}\right) \\
0(\text { otherwise })
\end{array}\right.
\end{gathered}
$$

We remark that this model is based on the following assumption.

\section{Assumption 1.}

1.) For any $i \in\{1,2, \cdots, n\}, \beta_{i}, \gamma_{i}$, and $v_{i}$ are positive constants.

2.) For any $i \in\{1,2, \cdots, n\}, S_{i}, I_{i}$, and $R_{i}$ are nonnegative.

3.) $p, t_{0}$, and $t_{1}$ are positive constants.

4.) For any $i, j \in\{1,2, \cdots, n\}, T_{i, j}$ is a positive constant.

5.) For any $i \in\{1,2, \cdots, \mathrm{n}\}, \sum_{\mathrm{j}=1}^{n} T_{\mathrm{i}, \mathrm{j}}=1$.

Assumption 1 1.) to 4.) refer to the biological validity while assumption 15 .) expresses a group of people that are in any group in the daytime. The overall diagram of the transition is shown in Fig. 1.

\section{The Basic Reproduction Number}

The basic reproduction number using next-generation matrix is calculated in this section ${ }^{(3,4)}$. Let $\boldsymbol{I}$ be equal to $\left(I_{1}, I_{2}, \cdots, I_{n}\right)^{T}$, and we assume disease-free state, for any $i \in\{1,2, \cdots, n\}$ with $S_{i}=N_{i}$. Thus, $d \boldsymbol{I} / \mathrm{dt}$ is given by the following equation:

$$
\frac{d \boldsymbol{I}}{d t}=(F-V) \boldsymbol{I}
$$

Matrices $F$ and $V$ are defined as follows:

$$
\begin{gathered}
F=\left[\frac{1}{2} \beta_{j}\left(N_{i} \sum_{k=1}^{n} \frac{T_{j, k} T_{i, k}}{N_{k}^{d}}+\delta_{i, j}\right)\right]_{i, j} \\
V=\left[\gamma_{i} \delta_{i, j}\right]_{i, j}
\end{gathered}
$$

where $\delta$ denotes the Kronecker delta. From $F$ and $V$, we calculate the next-generation matrix denoted by $K$. The basic reproduction number $R_{0}$, which is given by the spectral radius of $K$ expressed as the following equation ${ }^{(3)}$ :

$$
K=F V^{-1}=\left[\frac{\beta_{j}}{2 \gamma_{j}}\left(N_{i} \sum_{k=1}^{n} \frac{T_{j, k} T_{i, k}}{N_{k}^{d}}+\delta_{i, j}\right)\right]_{i, j}
$$

basic reproduction number refers to the average number of secondary cases due to primary cases into a totally susceptible population.

In addition, we assume the "passive reproduction number" for each group and the "reproduction number" for each group. Consequently, the following definitions and theorems are obtained.

\section{Definition 1.}

The passive reproduction number for group $i$ is defined as the sum of the average number of the secondary cases in group $i$ due to the primary cases.

\section{Theorem 1.}

The passive reproduction number for group $i$ can be calculated using the following equation:

$$
P_{i}=\sum_{j=1}^{n} K_{i, j}
$$

\section{Proof 1.}

First, we examine the primary cases in group 1. The number of primary cases in group 1 at time $t$ is expressed as equation (12), which is obtained from $d I_{1} / d t=-\gamma_{1} I_{1}$ :

$$
I_{1}=e^{-\gamma_{1}} I_{1}(0)
$$

where $I_{1}(0)$ denotes the number of the primary cases in group 1 at time 0 . Now, we examine the average number of the secondary cases in group $i$ caused by primary cases in group 1 . The integration of $F_{i, 1} I_{1}$ from 0 to infinity gives the total number of the secondary cases in group $i$ due to the primary cases in group 1 . Thus, we get the following equation:

$$
\int_{0}^{\infty} F_{i, 1} I_{1}=\frac{F_{i, 1}}{\gamma_{1}} I_{1}(0)
$$

From equation (13), we obtain the following equation, which is the average number of secondary cases:

$$
P_{i, 1}=\frac{F_{i, 1}}{\gamma_{1}}
$$

Furthermore, the average number of the secondary cases in group $i$ resulting from primary cases in group $n$ is expressed as the following equation:

$$
P_{i, n}=\frac{F_{i, n}}{\gamma_{n}}
$$

The passive reproduction number for group $i$ is the finite sum from $P_{i, 1}$ to $P_{i, n}$. Consequently, we obtain this equation below:

$$
P_{i}=\sum_{j=1}^{n} P_{i, j}=\sum_{j=1}^{n} \frac{F_{i, j}}{\gamma_{j}}=\sum_{j=1}^{n} K_{i, j}
$$




\section{Definition 2.}

The reproduction number for group $i$ defined as the sum of the average number of the secondary cases caused by the primary cases in group $i$.

\section{Theorem 2.}

The reproduction number for group $i$ can be calculated as the following equation:

$$
R_{0}^{i}=\sum_{j=1}^{n} K_{j, i}
$$

\section{Proof 2.}

We investigate the primary cases in group $i$. The number of the primary cases in group $i$ at time $t$ is expressed as equation (18), which is obtained from the following equation $d I_{i} / d t=-\gamma_{i} I_{i}$ :

$$
I_{i}=e^{-\gamma_{i}} I_{i}(0)
$$

where $I_{i}(0)$ denotes the number of the primary cases in group $i$ at time 0 . Now, we examine the average number of secondary cases in group 1 . The integration of $F_{1, i} I_{i}$ from 0 to infinity gives the total number of secondary cases in group 1. Thus, the following equation is obtained:

$$
\int_{0}^{\infty} F_{1, i} I_{1}=\frac{F_{1, i}}{\gamma_{i}} I_{i}(0)
$$

By utilizing equation (19), we obtain the equation below, which is the average number of secondary cases:

$$
R_{0}^{i, 1}=\frac{F_{1, i}}{\gamma_{i}}
$$

Similarly, the average number of secondary cases in group $n$ due to the primary cases in group $i$ is expressed as the following equation:

$$
R_{0}^{i, n}=\frac{F_{n, i}}{\gamma_{i}}
$$

The reproduction number for group $i$ is the finite sum from $R_{0}^{\mathrm{i}, 1}$ to $R_{0}^{\mathrm{i}, n}$. Thus, we obtain the equation below:

$$
R_{0}^{i}=\sum_{j=1}^{n} R_{0}^{i, j}=\sum_{j=1}^{n} \frac{F_{j, i}}{\gamma_{i}}=\sum_{j=1}^{n} K_{j, i}
$$

\section{Analysis of the Epidemic}

\subsection{Parameter Estimation}

In this section, we describe the method of model parameters estimation to analyze epidemic. Equation (23) is an estimation of error function:

$$
\begin{aligned}
\operatorname{Error}(\theta) & =\sum_{i=1}^{n} E_{i}(\theta) \\
E_{i}(\theta) & =\sum_{t=1}^{m}\left(I_{i}^{c, \text { data }}(t)-I_{i}^{c, \text { model }}(t)\right)^{2}
\end{aligned}
$$

where $\theta, n, m, I_{i}^{c, \text { data }}$ and $I_{i}^{c, \text { model }}$ denote the parameter set of infection and recovery rate, the number of the groups, the number of data, the actual cumulative of the infectious in group $i$, and the cumulative of infectious in group $i$ obtained from the epidemic model, respectively. Additionally, as not everyone is at risk for infection, we estimate $\alpha_{i}$ that is the ratio of the population and people at risk of infected. In other words, the product of $\alpha_{\mathrm{i}}$ and population in group $i$ is initial value of susceptible.

We use the Nelder-Mead method to estimate the parameters. This method does not use the derivatives of the objective function.

\subsection{Experiments}

The settings and experimental environment for estimation are described in this section. We give a number to the following cities: Tokyo, Kanagawa, Saitama, Chiba, Tochigi, Gunma, and Ibaraki which denote from group 1 to 7 , respectively. In addition, we use the data of the number of influenza patients from the 40th week in 2017 to the 22nd week in 2018 published by the National Institute of Infectious Disease ${ }^{(5)}$. The transfer rate of the outflow population is obtained from the national census in $2015 \mathrm{~s}$ (6) and set the vaccination rate to assumption 2 .

\section{Assumption 2.}

1.) People vaccinated are located in proportion to the population in each group.

2.) $v_{i}$ satisfies $S_{i}\left(\mathrm{t}_{0}\right)=C \exp \left(-v_{i} t_{0}\right)$ and $S_{i}\left(t_{1}\right)=$ $\left(1-r_{i}\right) S_{i}\left(\mathrm{t}_{0}\right)=\operatorname{Cexp}\left(-v_{i} t_{1}\right)$ if no infection occurs.

$r_{i}$ and $C$ are the ratio of the vaccination in group $i$ and constant, respectively. First, we calculate $r_{i}$ using the following equation:

$$
\begin{array}{r}
r_{i}=\frac{\text { vaccinated in Japan }}{\text { population in Japan }} \\
\times \frac{\text { population in group } i}{\text { population in Japan }}
\end{array}
$$

The data for the vaccination rate is obtained from the Ministry of Health, Labour, and Welfare in Japan ${ }^{(7)}$. Next, we calculate $v_{i}$ by applying assumption 22 .) and equation (24), as follows, 
Table 1. Transfer Rate.

\begin{tabular}{c|c|c|c|c|c|c|c}
\hline & 1 & 2 & 3 & 4 & 5 & 6 & 7 \\
\hline 1 & 0.9655 & 0.0172 & 0.0104 & 0.0061 & 0.0002 & 0.0001 & 0.0005 \\
\hline 2 & 0.1155 & 0.8810 & 0.0002 & 0.0002 & 0.0001 & 0.0001 & 0.0001 \\
\hline 3 & 0.1314 & 0.0039 & 0.8514 & 0.0059 & 0.0014 & 0.0040 & 0.0020 \\
\hline 4 & 0.1191 & 0.0042 & 0.0067 & 0.8632 & 0.0006 & 0.0006 & 0.0056 \\
\hline 5 & 0.0088 & 0.0001 & 0.0060 & 0.0020 & 0.9621 & 0.0118 & 0.0091 \\
\hline 6 & 0.0062 & 0.0001 & 0.0140 & 0.0020 & 0.0083 & 0.9599 & 0.0095 \\
\hline 7 & 0.0241 & 0.0001 & 0.0060 & 0.0152 & 0.0075 & 0.0008 & 0.9395 \\
\hline
\end{tabular}

Table 2. Vaccine parameters

\begin{tabular}{c|c|c}
\hline Symbol & Description & Value \\
\hline$p$ & Disease prevention rate & 0.9 \\
\hline$t_{0}$ & Start of vaccination & 1 \\
\hline$t_{1}$ & End of vaccination & 10 \\
\hline
\end{tabular}

Table 3. Vaccination rate

\begin{tabular}{c|c|c|c|c|c|c|c}
\hline Group & 1 & 2 & 3 & 4 & 5 & 6 & 7 \\
\hline$v_{i}\left(\times 10^{-4}\right)$ & 14.8 & 10.0 & 0.796 & 0.681 & 0.216 & 0.216 & 0.319 \\
\hline
\end{tabular}

Table 4. Population and initial values I, and R

\begin{tabular}{c|c|c|c}
\hline & Population & $\mathrm{I}$ & $\mathrm{R}$ \\
\hline 1 & 13515000 & 54 & 0 \\
\hline 2 & 9126000 & 68 & 0 \\
\hline 3 & 7267000 & 42 & 0 \\
\hline 4 & 6223000 & 35 & 0 \\
\hline 5 & 1974000 & 6 & 0 \\
\hline 6 & 1973000 & 3 & 0 \\
\hline 7 & 2917000 & 10 & 0 \\
\hline
\end{tabular}

$$
v_{i}=\frac{-\ln \left(1-r_{i}\right)}{t_{1}-t_{0}}
$$

We also set the disease prevention rate by the vaccination assumed value and the vaccination period from October to December in Japan. Tables 1, 2, 3, and 4 present the transfer rates (the all at row $i$ and column $j$ denotes the transfer rate from group $i$ to $j$ ) vaccine parameters vaccination rates, and population, initial value of $\mathrm{I}$, and $\mathrm{R}$, respectively.

The experiments were conducted using MATLAB R2019a. We used ode45 to solve the simultaneous differential equations and fminsearch to estimate the parameters.

\subsection{Results of the Estimation}

Table 5 shows the estimated values of infection rates for each group. The top row demonstrates the group numbers, the second row illustrates the infection rates. Fig. 2 depicts the plot of the cumulative of the infectious from the model using the estimated values and data. Fig. 3 depicts the plot of the sum of the infectious from model and data. Lines and circles in figs. 2 and 3 denote the numbers of the infectious from the model and data, respectively. Table 6 demonstrates the reproduction numbers for each group using the estimated values. The top row illustrates the group numbers, the second

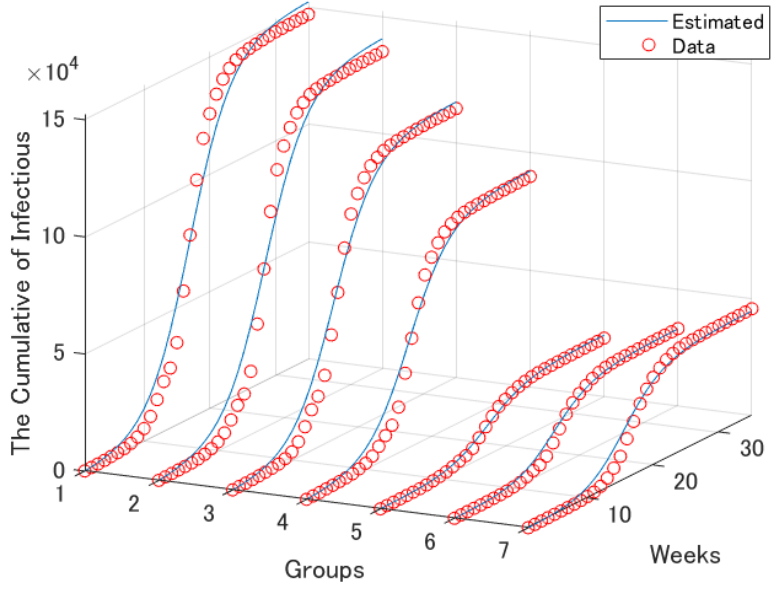

Fig. 2. The plot of the infectious people

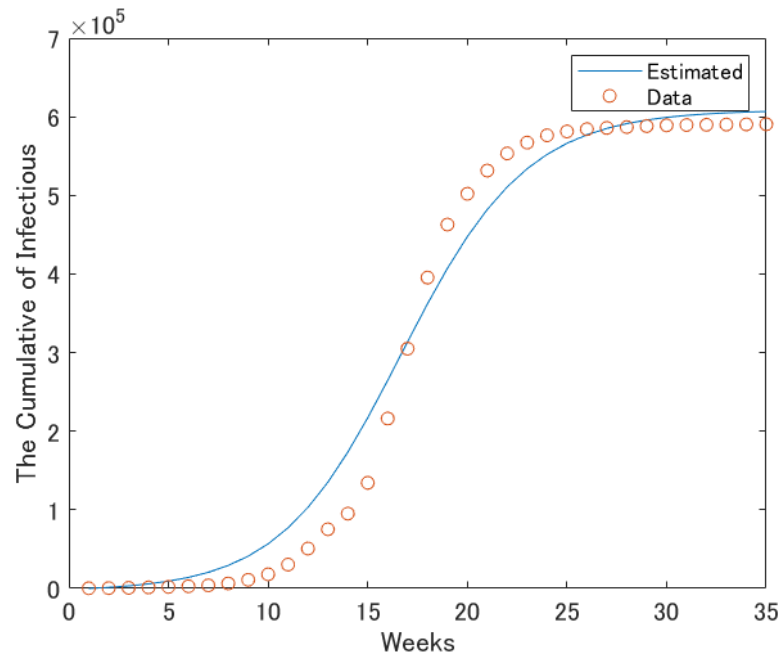

Fig. 3. The plot of the sum of infectious

Table 5. The estimated parameters

\begin{tabular}{c|c|c|c|c|c|c|c}
\hline Group & 1 & 2 & 3 & 4 & 5 & 6 & 7 \\
\hline$\beta_{i}$ & 0.744 & 0.751 & 0.753 & 0.752 & 0.621 & 0.733 & 0.755 \\
\hline$\gamma_{i}$ & 0.728 & 0.700 & 0.673 & 0.688 & 0.582 & 0.678 & 0.687 \\
\hline$\alpha_{i}$ & 0.118 & 0.145 & 0.114 & 0.116 & 0.106 & 0.118 & 0.100 \\
\hline
\end{tabular}

Table 6. Reproduction number for each group.

\begin{tabular}{c|c|c|c|c|c|c|c}
\hline Group & 1 & 2 & 3 & 4 & 5 & 6 & 7 \\
\hline$P_{i}$ & 1.12 & 1.07 & 1.10 & 1.07 & 1.05 & 1.07 & 1.08 \\
\hline$R_{0}^{i}$ & 1.02 & 1.07 & 1.12 & 1.08 & 1.07 & 1.08 & 1.10 \\
\hline
\end{tabular}

row shows the passive reproduction numbers of each group, and the bottom row demonstrates the reproduction numbers of each group. Furthermore, the basic reproduction number is equal to 1.08 .

\section{Suppression of the Epidemic}

This section discusses how to suppress epidemic by taking some preventive measures, such as vaccination and individual efforts against infection. First, we examine how to 


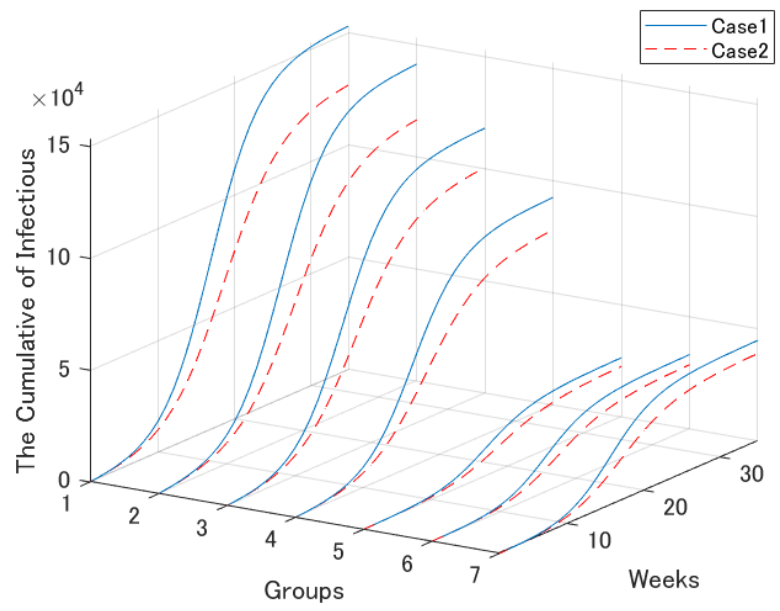

(a) The comparison of each group

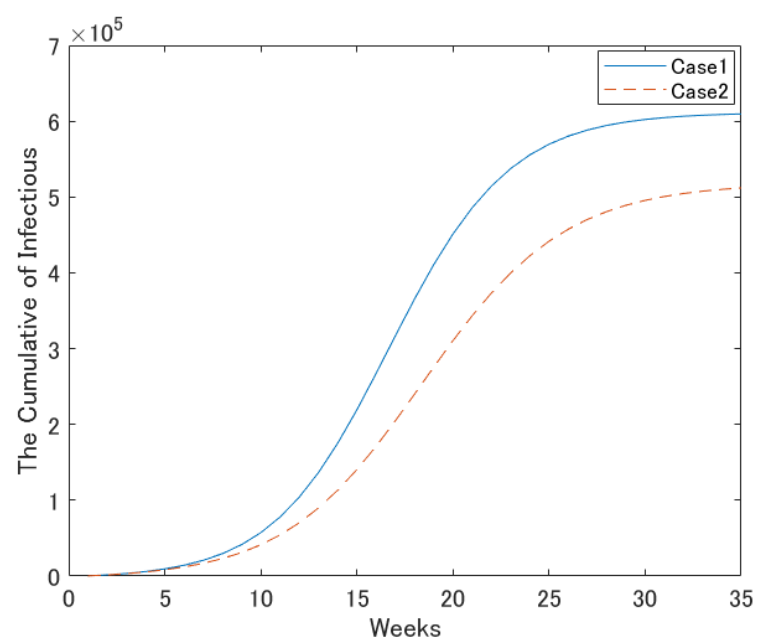

(b) The comparison of the total

Fig. 4. The comparison of the number of infectious with and without preventive efforts

Table 7. Ratio of the number of infectious

\begin{tabular}{l|c|c|c|c|c|c|c|c}
\hline Group & All & 1 & 2 & 3 & 4 & 5 & 6 & 7 \\
\hline Peak & 0.72 & 0.71 & 0.71 & 0.73 & 0.72 & 0.74 & 0.75 & 0.74 \\
\hline Total & 0.84 & 0.83 & 0.82 & 0.85 & 0.84 & 0.85 & 0.86 & 0.87 \\
\hline
\end{tabular}

reduce the infection rate by the individuals who take care of preventing infection. The infection rate slightly decreases because it is affected by the characteristic of the pathogen. Therefore, we assumed that the infection rate could be reduced by $1 \%$ and then multiplied $\beta_{i}$ by 0.99 . Now, we examine the effect of vaccination. We multiplied $r_{i}$ by 1.06 assuming that all the supplied vaccines have been used ${ }^{(8)}$.

Fig.4 depicts the comparison of the number of the infectious. The solid line depicts the plots of the results without preventive efforts (case 1) while the dash line depicts the plots of the results with efforts (case 2). Fig. 4(a)

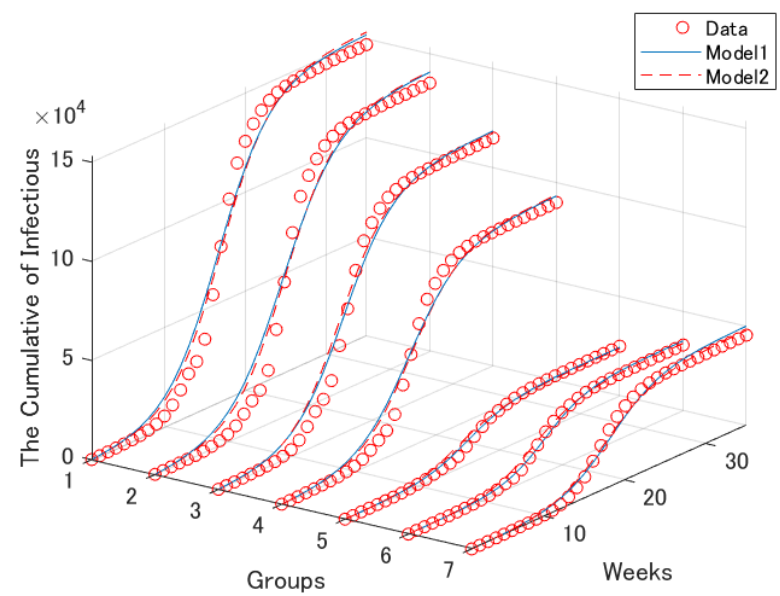

(a) The comparison between models 1 and 2

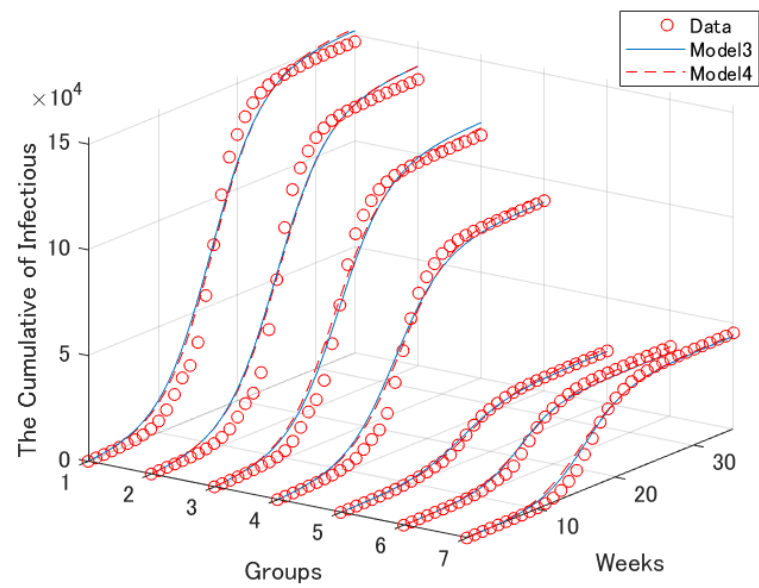

(b) The comparison between models 3 and 4

Fig. 5. The comparison of the estimation using differ at models

Table 8 . The settings and the results

\begin{tabular}{c|c|c|c|c}
\hline Model & 1 & 2 & 3 & 4 \\
\hline$T$ & Identity & Table 1 & Identity & Table 1 \\
\hline$v_{i}$ & 0 & 0 & Table 3 & Table 3 \\
\hline Error $\left(\times 10^{9}\right)$ & 8.88 & 7.70 & 9.00 & 8.48 \\
\hline
\end{tabular}

illustrates the comparison of each group between cases 1 and 2. Fig.4(b) demonstrates the comparison of the total between cases 1 and 2. Table 7 presents the ratios of the number of infectious. The top row denotes the group ID and "all" denotes the average of the whole group. The second row shows the ratios of the number of the infectious at peak time (week with largest difference) between cases 1 and 2. The bottom row demonstrates the ratios of the total number of the infectious (the cumulative number of the infectious at 35 th weeks) between cases 1 and 2 . 


\section{Discussion}

Our models and experimental results are discussed in this section, so far as the comparison of the errors between the SIR and our models. We label the SIR model, our model without vaccination, SIR model with vaccination, and our model as models 1, 2, 3, and 4, respectively and evaluate using equation (22). When estimating them using models 1 and 3 , we set the identity matrix as $T$ to treat our model as the SIR model, and if the model does not consider vaccination, we set $v_{i}$ as 0 . Table 7 shows the settings and the results of the estimation. The top row denotes the model number. The second and third rows demonstrate the settings of $T$ and $v_{i}$, respectively. The bottom row illustrates the errors using the estimated parameters and equation (22). Fig. 5 depicts the comparison between the SIR model and our models. Fig. 4(a) shows the comparison of the estimation between models 1 and 2. Circles, lines and dashed line denote the cumulative of the infectious from the data, model 1 and models 2, respectively. Fig. 4(b) depicts the comparison of the estimation between models 3 and 4 . Circles, lines and dashes line denote the numbers of the infectious from the data, model 3 and model 4, respectively. From table 8 , we observed that the estimation using our model is slightly better than that of the SIR model. However, the error is not so large that we should improve ours model.

The reproduction number and the suppression of an epidemic are other interesting points. In section 4, we obtained the basic reproduction number to be 1.08 while the reproduction numbers were obtained on the third row of table 6 . This shows that Tokyo is the least reproduction number. However, from the second row of table 6, Tokyo has a higher passive reproduction number when compared with others. This indicates that Tokyo was the most affected by primary cases from other prefectures. In addition, table 7 showed that, if vaccinations were adequately taken and individuals took adequate preventive measures against infection, it enables the number of the infected people at peak time to be suppressed by about $30 \%$ and the total number of the infected people could be suppressed by about $15 \%$.

\section{Conclusion}

This paper proposed an epidemic model with the person trip and analyzed real epidemics of influenza virus in the Kanto region in Japan from 2017 to 2018. The basic reproduction number was obtained to be 1.08 due to parameters' estimation and calculation. When adequate vaccinations were taken and adequate preventive measures were taken against infection by people, the number of infected people was suppressed by about $30 \%$ at the peak time and the total infected people could be suppressed by about $15 \%$. We continue to improve this model to apply it to other infectious diseases.

\section{Acknowledgment}

This work was supported by JSPS KAKENHI Grant Number JP17K00138.

\section{References}

(1) Emilia Vynnycky and Richard G White: "An introduction to infectious disease modeling", Oxford University Press, 2010.

(2) Takehisa Yamamoto, Yoko Hayama, and Toshiyuki TsuTsui: "Brief evaluation of transmission dynamics of ebola virus disease using mathematical model", Journal of Veterinary Epidemiology, Vol. 19, No. 1, pp. 48-54, 2015.

(3) O. Diekman, J. A. P. Heesterbeek, and M. G. Roberts: "The Construction of next-generation matrices for compartmental epidemic models", Journal of the Royal Society Interface, Vol. 7, No. 47, pp. 873-885, 2010.

(4) P. van den Driessche, and James Watmough: "Reproduction numbers and sub-threshold endemic equilibria for compartmental models for disease transmission", Mathematical Bioscience, Vol. 180, pp. 29-48, 2002.

(5) Infectious Disease Weekly Report, National Institute of Infectious Disease, https://www.niid.go.jp/niid/en/idwre.html, Accessed 6/18/2018.

(6) Summary of the results and statistical tables: Statistics Bureau Ministry of Internal Affairs and Communications, https://www.stat.go.jp/english/data/kokusei/2015/sum mary.html, Accessed 7/6/2018.

(7) The number of vaccination implementation persons of the fixed period, Ministry of Health, Labour and Welfare, https://www.mhlw.go.jp/topics/bcg/other/5.html, Accessed 8/14/2018

(8) About supply of influenza vaccine of 2018/2019 season: Ministry of Health, Labour and Welfare, https://www.mhlw.go.jp/content/10601000/000352296. pdf, Accessed 12/3/2018. 